Supporting Information for:

\title{
Catalytic enhancement of $\mathrm{CO}$ oxidation on $\mathrm{LaFeO}_{3}$ regulated by Ruddlesden-Popper stacking faults
}

Reut Bornovski ${ }^{1}$, Liang-Feng Huang ${ }^{2,3}$, Eswaravara Prasadarao Komarala ${ }^{1}$, James M. Rondinelli ${ }^{2}$, and Brian A. Rosen ${ }^{1 *}$

1) Department of Materials Science and Engineering, Tel Aviv University, Ramat Aviv, 69987001, ISRAEL

2) Department of Materials Science and Engineering, Northwestern University, Evanston Illinois 60208-3108, USA

3) Key Laboratory of Marine Materials and Related Technologies, Key Laboratory of Marine Materials and Protective Technologies of Zhejiang Province, Ningbo Institute of Materials Technology and Engineering, Chinese Academy of Sciences, Ningbo 315201, CHINA

*corresponding author: barosen@post.tau.ac.il 

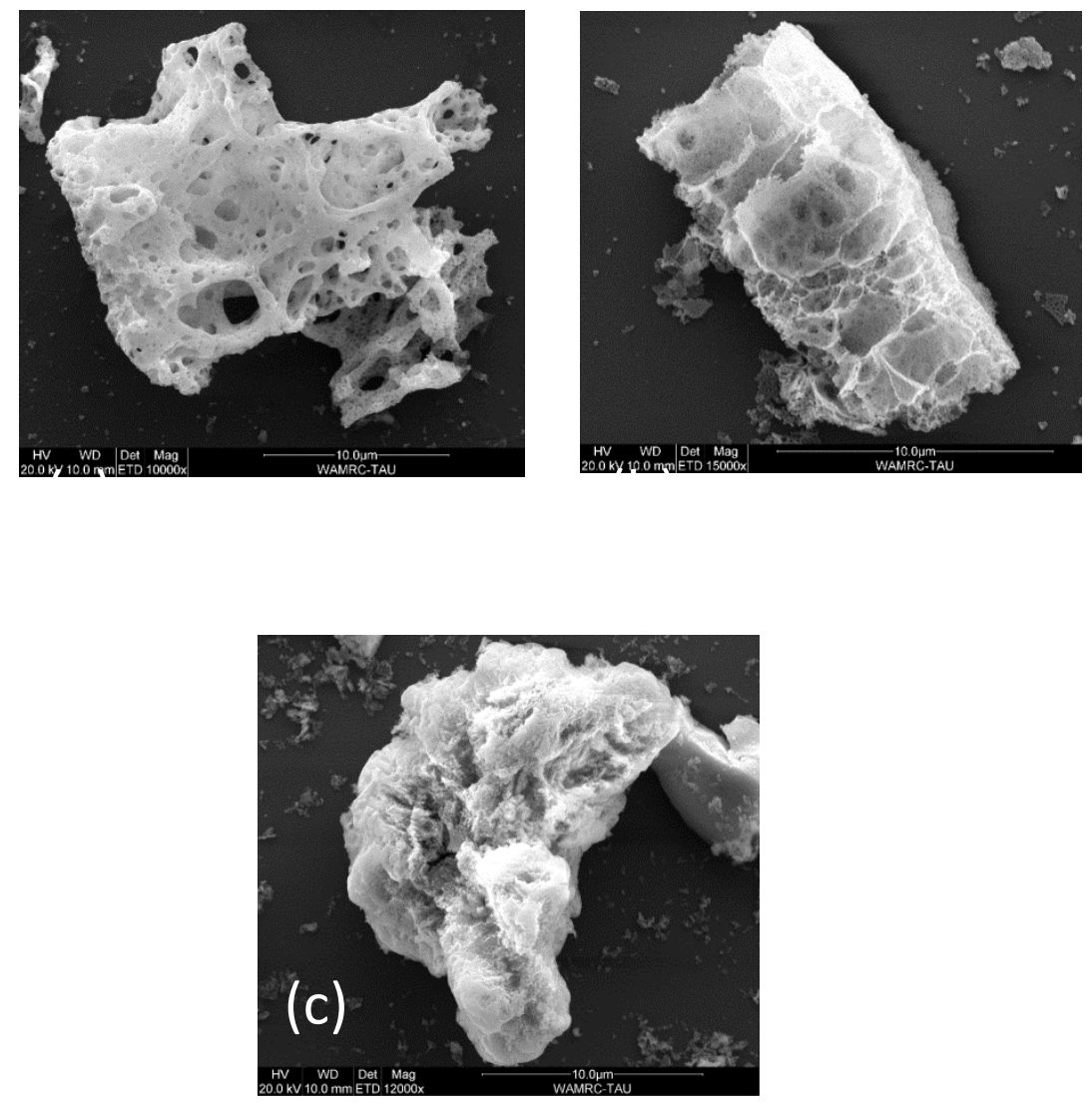

Figure S1: SEM images showing the morphology of (a) cs-LF, (b) sg-LF, and (c) cp-LF.

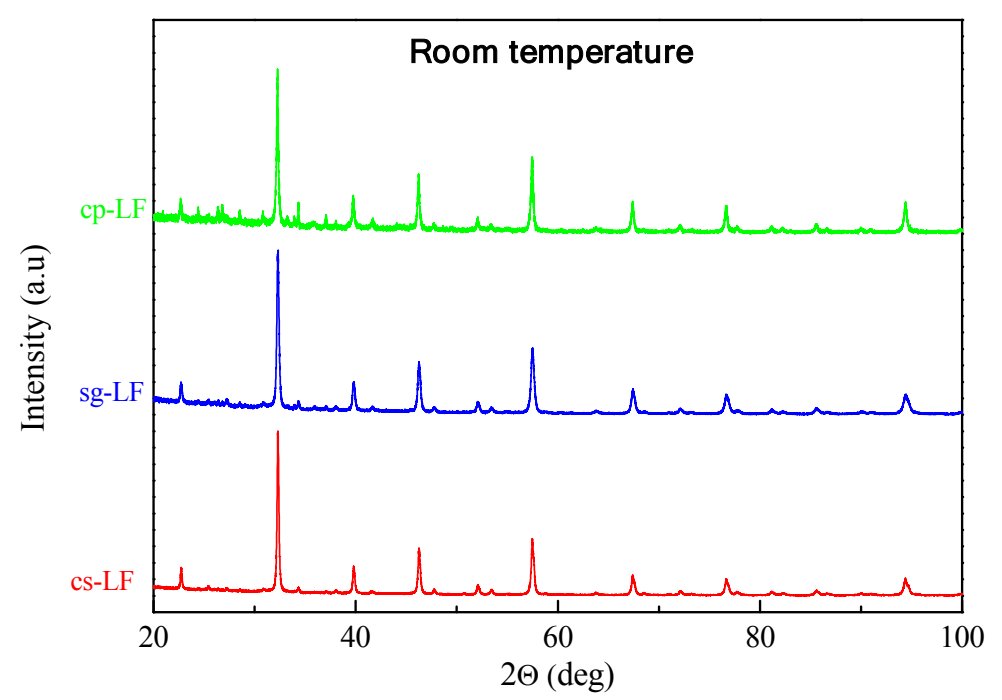




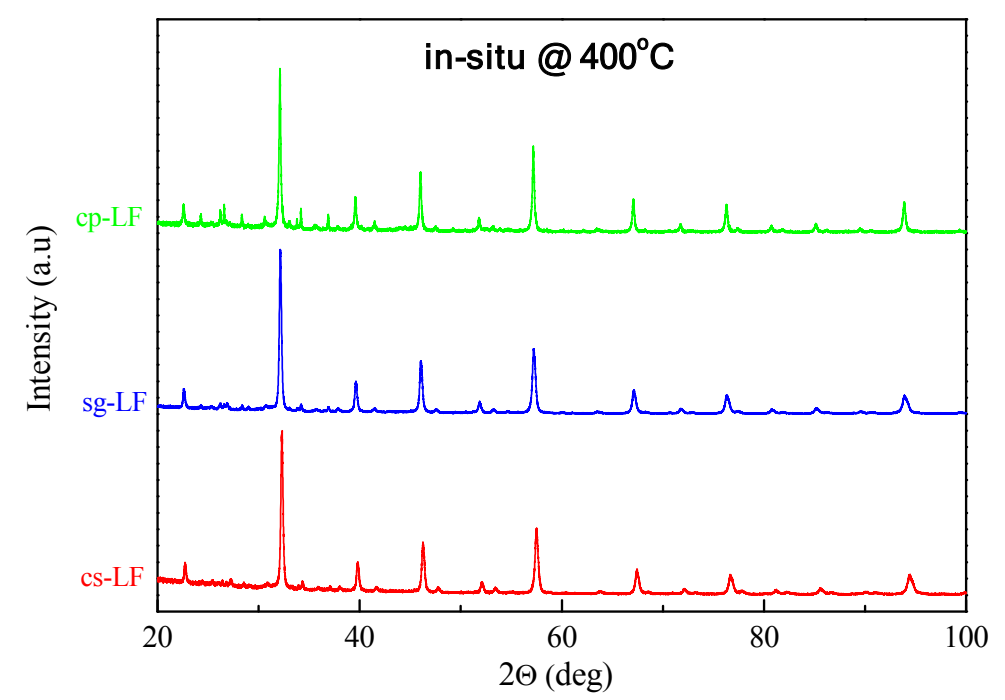

\begin{tabular}{|c|c|c|c|c|}
\hline & & cs-LF & sg-LF & cP-LF \\
\hline Lattice parameter - a & $[\AA]]$ & 5.570 & 5.563 & 5.568 \\
\hline Lattice parameter - b & $[\AA]]$ & 7.861 & 7.865 & 7.858 \\
\hline Lattice parameter - c & $[\AA]]$ & 5.562 & 5.562 & 5.558 \\
\hline Cell volume & {$\left[\AA^{3}\right]$} & 243.512 & 243.352 & 243.162 \\
\hline
\end{tabular}

Figure S2: XRD patterns and fitted room-temperature lattice parameters for assynthesized cs-LF, sg-LF, and cp-LF. 


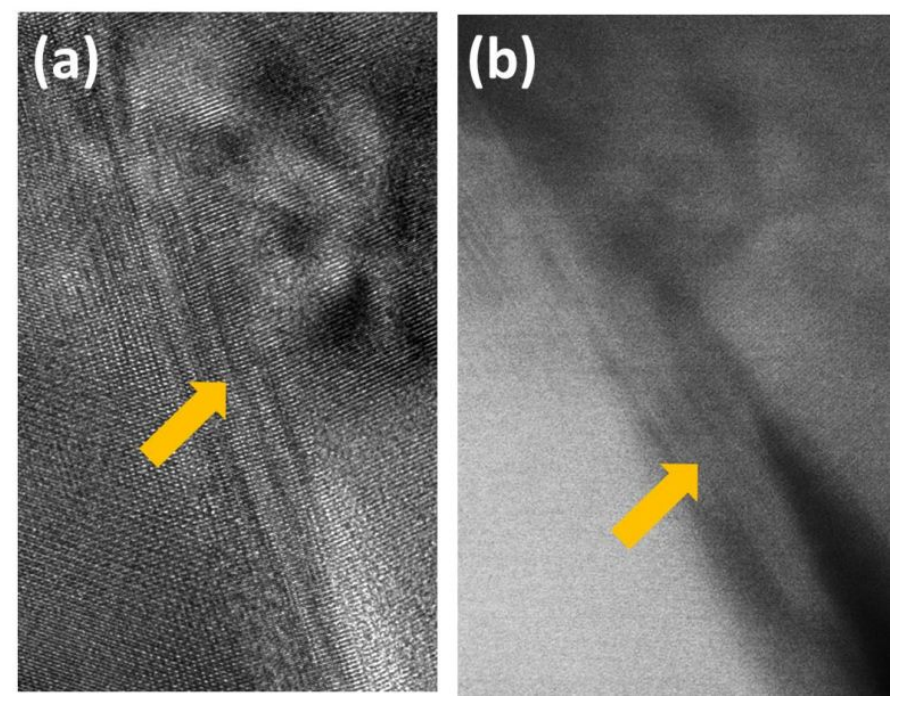

Figure S3: (a) Bright-field (BF) and (b) High-angle annular dark-field (HAADF) images of a faulted region near the surface of sg-LF.
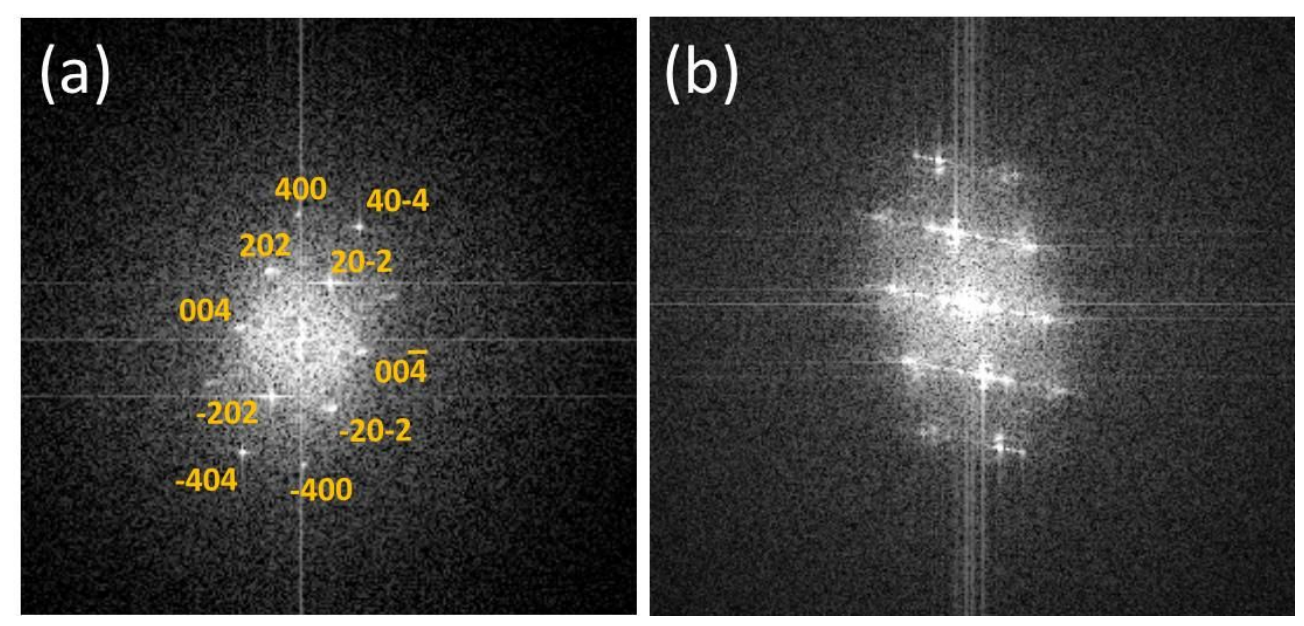

Figure S4: Fourier transformations of (a) pristine and (b) faulted regions in cp-LF using the TEM image shown in Figure 1c. 


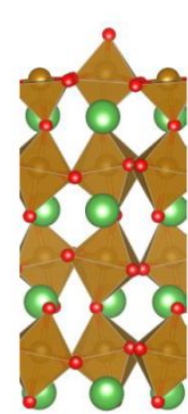

$\mathbf{0}$

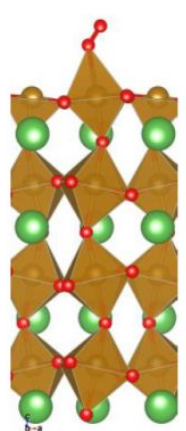

$\mathbf{O}_{2}$

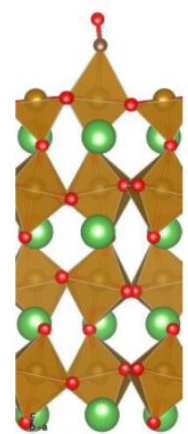

CO

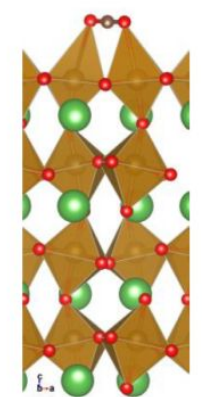

$\mathrm{CO}_{2}$

Figure S5: Configuration of $\mathrm{O}, \mathrm{O}_{2}, \mathrm{CO}$, and $\mathrm{CO}_{2}$ adsorption on pristine $\mathrm{FeO}_{2}-$ terminated $\mathrm{LaFeO}_{3}$.
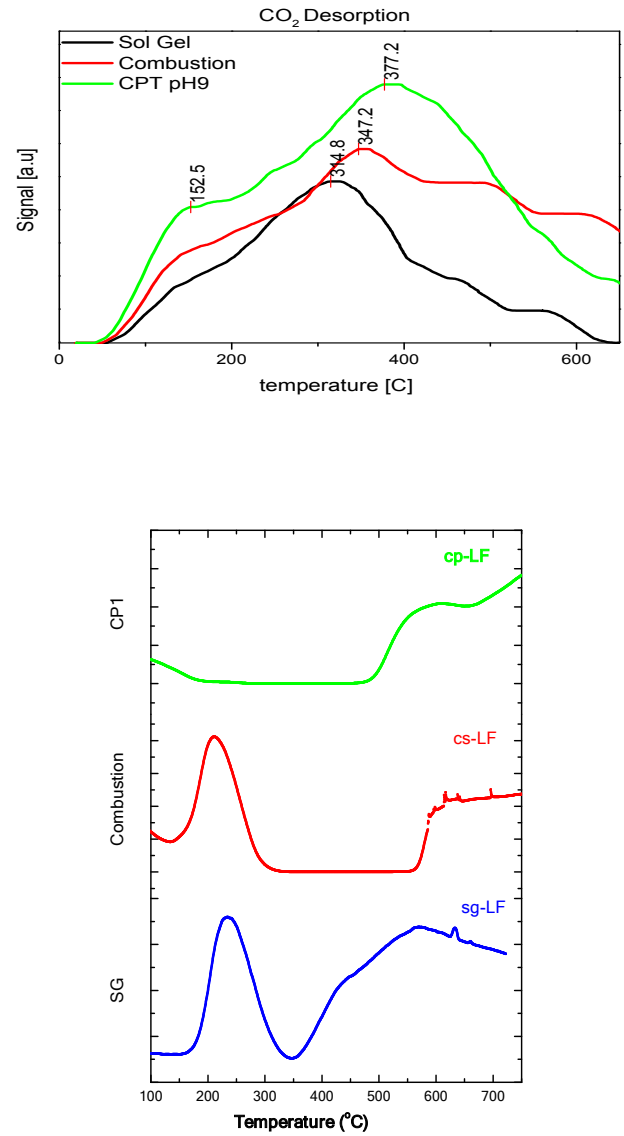

Figure S6: $\mathrm{CO}_{2}$ (top) and $\mathrm{O}_{2}$ (bottom) TPD on all samples 
S-5

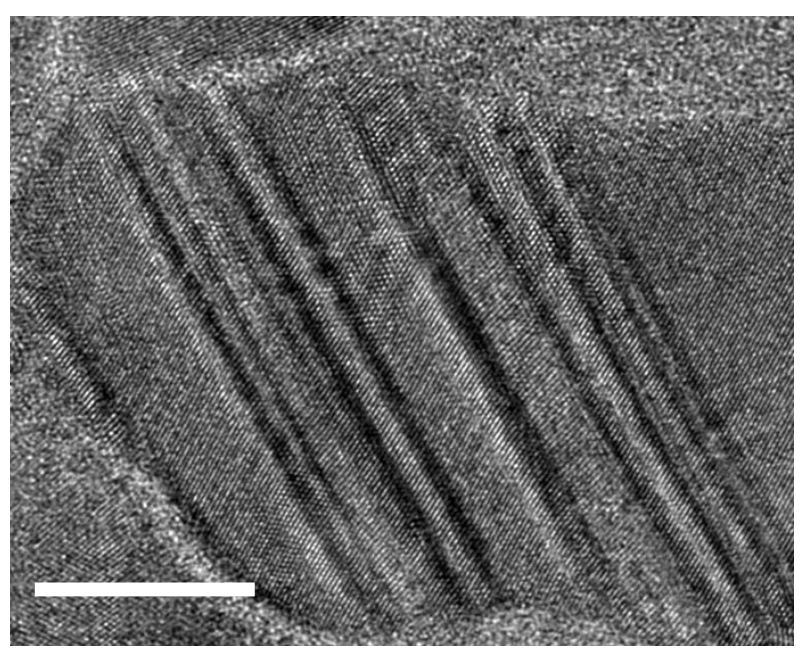

Figure S7: High-temperature TEM of cp-LF at $400^{\circ} \mathrm{C}$, used to confirm that the stacking faults are still present at the maximum reaction temperature in vacuum. Scale bar $10 \mathrm{~nm}$.

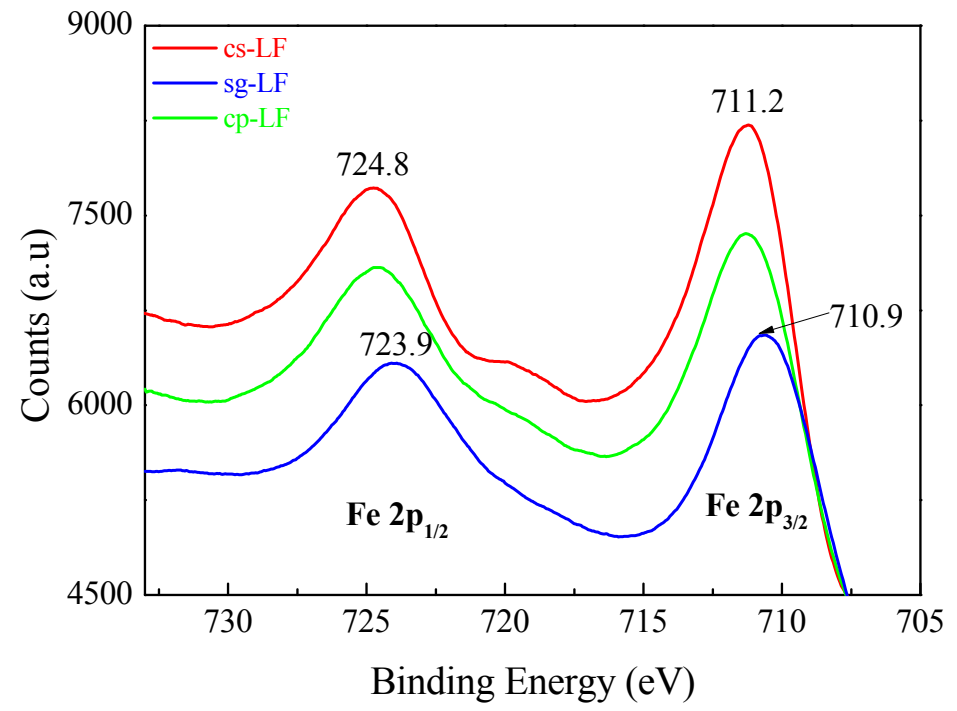


Figure S8: High-resolution XPS of the Fe 2p electron for as-prepared $\mathrm{LaFeO}_{3}$ using all three synthesis routes

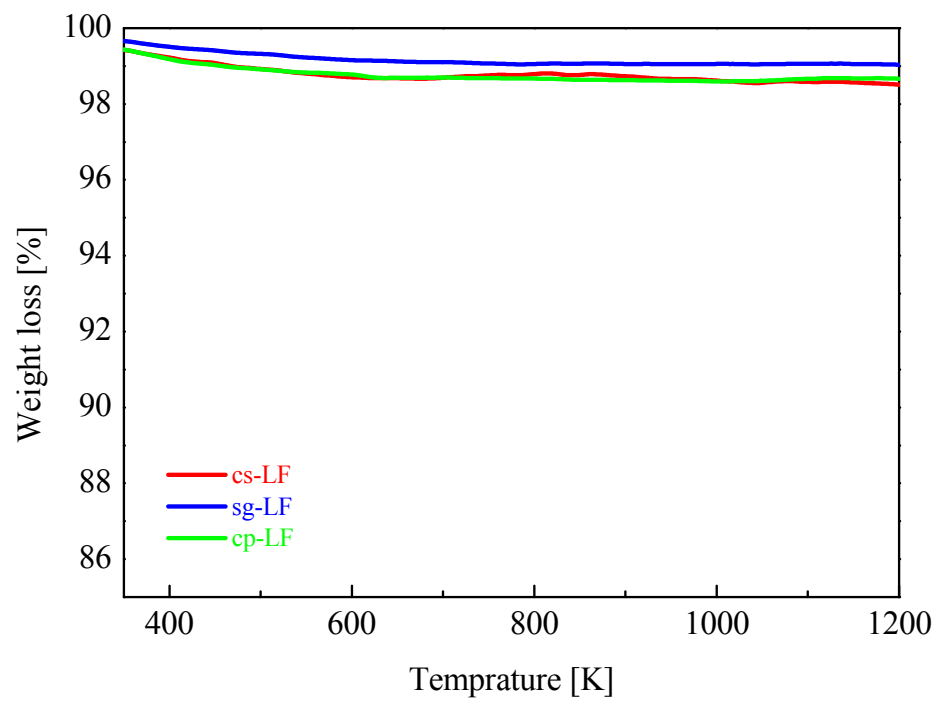

Figure S9: Thermographic analysis of all three $\mathrm{LaFeO}_{3}$ samples in a $20 \%$ oxygen atmosphere up to $1000^{\circ} \mathrm{C}$ using a heating rate of $5^{\circ} \mathrm{C} / \mathrm{min}$. 
